\title{
Strategies toward the development of an expert system for sensory evaluation testing and database management
}

\author{
C. L. KUESTEN \\ S. C. Johnson Wax, Racine, Wisconsin \\ and \\ M. R. MCLELLAN \\ Cornell University, Geneva, New York
}

\begin{abstract}
This paper presents design, knowledge representation, and implementation strategies aimed at the development of a knowledge system to guide and assist the sensory scientist in selection and implementation of appropriate test methods, statistical/graphical analyses, panel training, and data management. Particular emphasis is placed on how such a system can be used for panel management activities, such as screening/selecting panelists and performance monitoring. The system was designed with the capability of providing individualized graphic feedback during panel training sessions on discrimination and descriptive tasks. The prototype was built on a Macintosh computer using the Goldworks II expert system shell. Other software was interfaced and interacts with the system to provide statistical, graphical, and database support.
\end{abstract}

Sensory evaluation as a scientific discipline has rapidly developed over the past 50 years. Measurement of sensory properties and their relationship to product quality and consumer acceptance has steadily gained recognition as a unique function within academic and business environments. Significant advances are being made in the area of sensory evaluation due to application of knowledge of human behavior and a more systematic and professional approach to testing. The sensory professional should be familiar with sensory test methodology and command a background in food science, human perception and behavior, statistics, and experimental design. Discussion at the 1989 Institute of Food Technologists' Sensory Symposium revealed concern regarding limited sensory and statistical competence. The problems confronting the sensory professional are (1) determining test objective(s), (2) selection of the appropriate test method(s), (3) adequate panel training, and (4) correct analysis and interpretation of data.

Statistical expertise is often sought as sensory professionals make heavy use of statistics and experimental design. As more and more computing power becomes available to users of statistics, it becomes a concern to statisticians that these statistical tools be used properly. Several statistical expert system examples have been developed to address this concern. REX and DEXPERT are two examples. REX is a regression expert system writ-

This work was supported by the New York State Agricultural Experiment Station, Geneva, NY. Appreciation is extended to the faculty, staff, and students who participated as contributors and panel members in the studies. Correspondence should be addressed to $C$. Kuesten, Research Services, S. C. Johnson Wax, Racine, WI 53403. ten by Gale and Pregibon at Bell Labs (Pregibon \& Gale, 1984). DEXPERT is an expert system for the design of experiments produced by researchers at General Motors (Lorenzen \& Truss, 1990). These systems have been designed to provide a general statistical guide with no specific targeted domain for use. The present effort, in contrast, is devoted to the development of an expert system specific to the domain of sensory evaluation research.

\section{Expert Systems}

The advent of artificial intelligence (AI) and expert systems has become one of the fastest growing segments of the computer industry. Numerous books and articles have been published summarizing concepts and techniques for AI and expert systems (e.g., Frenzel, 1987; Gale, 1986; Hayes-Roth, Waterman, \& Lenat, 1983; McLellan, 1989; Waterman, 1985). Moreover, Harmon (1987) has proposed that expert systems will rapidly proliferate to serve as intelligent job aids to promote employee performance. Professor Edward Feigenbaum defines an expert system as "an intelligent computer program that uses knowledge and inference procedures to solve problems that are difficult enough to require significant human expertise for their solution" (Harmon \& King, 1985, p. 5).

An expert system consists of a knowledge base, an inferencing system that can use the knowledge in problem solving, and the user interface (the input-output mechanism for facts, questions, explanations, and solutions). The knowledge base may contain facts, beliefs, rules, procedures, and practical shortcuts pertinent to the problem domain. The user can choose from among predicate calculus, lists, frames, and rules to represent this knowledge. The function of the inference engine is hypothesis prov- 
ing: various rules are fired to drive and ultimately prove or disprove the hypothesis.

An expert system shell is a program that functions to provide a framework in which to express knowledge. It is a software package with a collection of tools that enable users to develop expert systems. Each shell features a variety of capabilities and requires varying levels of user expertise and sophistication. All shells contain a knowledge base, an inference engine, and a user interface as discussed above.

\section{Objective}

The major objective for this research effort was to provide computerized instruction and assistance for a select subset of sensory evaluation test methods routinely used in panel development. This would include design and deliverance of an interactive system combining the domains of sensory evaluation and statistics to assist and/or facilitate the job of the sensory scientist through method selection, computer-aided design, statistical/graphical support, data management capabilities, and report generation. Because less than adequate panel training and monitoring effectively diminishes the potential quality of sensory data, a major emphasis has been directed toward delivering a tool that would aid in evaluating panelist performance and panel monitoring. Traditional sensory training focuses on roundtable discussions where panelists are trained on which attributes to score and how to use the scale. Biases are introduced in this group context, and the roundtable approach may distort natural differences in panelists' perceptions. On the other hand, no training makes it difficult for panelists to give precise scores. To retain the natural differences between individuals, a nonstructured scale and individualized training would be ideal (Naes, 1990). With the use of computer graphics and expert systems, this approach is feasible. The expert system has been designed to maintain up-to-date records of a panelist's discrimination and descriptive abilities, make decisions based on the performance levels achieved, determine the effectiveness of descriptive terms, and track products/samples over time.

\section{Software/Hardware Considerations}

Several commercially available shells were reviewed prior to selection of the Goldworks II (Goldhill Computers, Inc., Cambridge, MA) expert system shell. The selection of this shell was based on performance capabilities, cost considerations, extendibility, and ease of integration with other software. Statistical and graphics packages and database software available for the Macintosh computer were reviewed. SYSTAT (SYSTAT, Inc., Evanston, IL) was integrated to provide access to statistical and graphical analysis. Foxbase (Fox Software, Inc., Perrysburg, $\mathrm{OH}$ ) was chosen to provide database capabilities.

\section{Knowledge Acquisition}

Knowledge acquisition (the task of obtaining knowledge from human experts, case histories, or reference sources and formalizing it so that it can be included in the expert system knowledge base) proves to be one of the most dif- ficult aspects of developing an expert system. Numerous approaches have been developed to acquire knowledge as a means of developing structures, rules, and procedures for emulating the relevant expertise. Kahn, Nowlan, and McDermott (1985) offer knowledge acquisition strategies useful for diagnostic expert systems that proved useful toward the development of our prototype. These strategies are described in terms of symptoms, diagnosable events, and background conditions consequent to or affecting the occurrence of causes or hypotheses. Another structured approach we used is the knowledge matrix and the rule table (Braun, 1989). Facts of the domain are displayed in a matrix and through propositional logic converted to a rule table. IF-THEN statements are extracted from the rule table toward development of the rule base. These tools help assemble knowledge systematically, eliminate duplications, and assure reasonable completeness and correctness of the knowledge base.

\section{Knowledge Representation Frames}

A part of the information within this system is structured in a network representation called a lattice, which is composed of frames and instances. An instance has the same slots as the associated frame but holds specific values in the slots. For example, different panelists can be represented by filling in slot values that serve to describe the attributes of that particular panelist. Each particular panelist is represented as an instance of the frame panelist.

Figure 1 illustrates the lattice designed for the sensory evaluation expert system prototype. The lattice shows the set of objects selected to make up the knowledge domain for sensory evaluation. Within the domain, we have represented test, panel, and panelist objects, and so on. Once the set of objects is determined, it is necessary to define the objects and their interactions to complete the knowledge representation of the domain. This involves utilizing the elementary components of knowledge: naming, describing, organizing, relating, and constraining the objects (Parsaye \& Chignell, 1988). The following discussion offers insight into the frame representation in Figure 1. Central to this representation scheme is the test frame. Completion of the information in the test frame

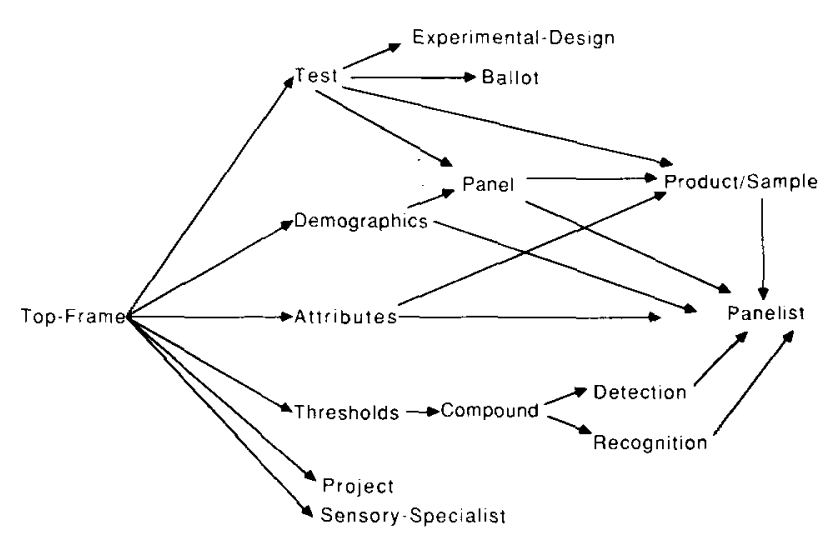

Figure 1. Lattice for sensory evaluation application. 
defines a new test instance for which data can be collected, analyses conducted, and reports generated. Other specific object instances (products, panelists, compounds, etc.) collectively form a test. Although there is some flexibility within the prototype, most of these objects, with the exception of the test design and analysis, must be created prior to defining a new test.

\section{Test Frame}

Because a given sensory evaluation test is defined by and composed of the other entities or objects defined in the system (panels, panelists, ballots, experimental designs, and analyses), the test frame is composed of slots to record which of these objects are involved in a given test, as well as specific details related to those objects. Many of the test frame slots are self-explanatory, holding names, text, dates, or numbers. Embedded within the scheme are association lists, which consist of a series of key/datum pairs; the key represents the classification code number assigned to the panelist for a test, and the datum holds the panelist's unique identification number. Management of test experimental design follows an objectoriented paradigm adapted from Oldford (1987).

\section{Panelist Frame}

The panelist frame itself holds only three slots to characterize a particular panelist: name, identification number, and panel. Because the panelist frame is a child frame of the demographics, attributes, and thresholds frames (as noted by arrows in Figure 1), the panelist frame will inherit all the slots contained by these frames as well. The panelist is characterized by not only a name, identification number, and panel membership, but also by demographic information, as well as performance information on attribute ratings and thresholds.

\section{Demographics, Attributes, and Thresholds Frames}

The demographics frame holds slots for all demographic items, such as age, preferences, and so forth. The attributes frame holds slots of all attributes contained within the attributes dictionary. Adding new attributes to the attribute dictionary automatically appends these attributes as slots into this frame. The thresholds frame holds slots of all compound objects represented within the system. Two frames inherit all the slots of the thresholds frame: the recognition frame and the detection frame. Values asserted to these slots will represent the specific types of thresholds for individual panelists.

\section{Panel Frame}

Panelists retain membership on one or more panels. The panel frame maintains selection and screening criteria for the panel, as well as dynamic lists of panelist status (selected, screened, qualified, in-training, or retraining). Panelists are added to or removed from these lists, depending on the criteria they hold or the performance they have demonstrated.

\section{Project and Product/Sample Frames}

The project frame is primarily used for maintaining records on projects. It includes name, number, and objective slots. The information then serves as a permanent record for this product or sample, and it may be later retrieved for inclusion in a test or for generating report summaries tracking a particular product. The product/ sample frame slots include commodity, description, name, and identification code.

\section{Compound Frames}

The slots contained within these frames are selfexplanatory. Note that the inheritance structure allows us to represent many compounds, each of which may exist in numerous products or samples, yet we can represent this concisely by completing the general information with the compound frame slots and further describing this compound as our needs dictate through inheritance for specific products/samples.

\section{Knowledge Representation: Rules}

Rules are used within this system to represent the domain knowledge and move the user as he/she interacts with the system from one menu or window to the next. Rules in conjunction with menus guide the user to sensory methods and offer a printed explanation of why certain methods were suggested. Data-driven rules are used within the system to effect decisions on panelist performance and panel membership. Examples are illustrated below. The rule base is easily extended, allowing the system to be refined and expanded.

\section{User Interface}

The user interface may be designed to interact with the user in a specified manner. Four screen layouts are presented to provide an understanding of how a part of this system has been designed for the test administrator.

Various buttons and edit fields allow the user to input information relevant to a particular test in the test screen. The user specifies the design by completing the information requested on the design screen. The user may search for, select, and/or screen panel members on the basis of demographics, attributes, thresholds, or performance criteria using the panel screen. Output may be generated to alert the test administrator of panelists with performance problems or abnormal test results relative to the rest of the panel. This facilitates a continuous performance check.

A ballot screen serves as a tool for ballot preparation and printing. Once a ballot is created and saved, it is permanently stored and may be used for future tests. The type of ballot currently available on the system is dependent on the test type selected by the test administrator. Currently, the prototype system supports the following sensory test methods: recognition tests, identification tests, paired comparison, triangle and duo-trio discrimination tests, a rank test, 7- or 9-point integer scales, line scales, and magnitude estimation scaling methods. In addition, 
the prototype can deliver an odor identification test with or without correct-response feedback.

Once a test has been completely defined, the test is ready for execution. Ballots may be either delivered to the screen or printed on paper. Samples are identified with unique three-digit code numbers, randomized appropriately across the panel, and delivered in appropriate order to the panelists. If the test is delivered via the computer, the test administrator has the option of providing feedback (either correct-response feedback or a graphic display generated by SYSTAT) to the panelists. Graphics produced in SYSTAT are available for displaying means and standard deviations by attribute. The test administrator also has the option of delivering all or part of the test in one sitting. Once data acquisition has been completed for a test, the data are parsed to an ASCII file and thereafter analyzed with SYSTAT. The SYSTAT command file is written by the system, calling in information as needed from the experimental design for that test. Further processing using LISP functions acquire information from the analysis outputs, such as means, standard deviations, $F$ values, and $p$ values, for report generation and maintaining performance records. Records are parsed to panel and individual panelist instances for updating performance records. This information in turn is appended to database structures created in Foxbase for permanent storage and retrieval.

\section{Panel Development}

Panelists are qualified for participation on sensory panels by performing at a certain level on discrimination and/or descriptive tests. To be considered eligible for further training, ASTM (1981) offers guidelines for screening panelists on the basis of results of a basic taste recognition test ( $\geq 70 \%$ correct requirement), an odor identification task (100\% correct), a rank test of the four basic tastes (sweet, sour, salty, and bitter; a Friedman Rank cutoff $p$ value of .05 is suggested), and a score of $\geq 60 \%$ on a discrimination task composed of a series of 24 triangle tests. Figure 2 illustrates how these decision criteria are represented as rules within the system using a backward chaining inferencing technique to qualify panelists for further training on a quality control panel. It should be noted that the default criteria (ASTM guidelines) may be modified by the user to establish more or less stringent screening criteria. Figure 3 illustrates a forward chaining inferencing technique to query the system on panelists who maintain a malic acid threshold of $10 \mathrm{ppm}$.

Typically, development efforts take a product through a number of modifications/improvements. The system has been designed to facilitate information retrieval. The power of the system lies in its ability to search, access, retrieve, and deliver this information in a convenient format to the user.

\section{Advantages/Disadvantages}

An expert system for the sensory professional may help maximize efficiency, accuracy, and consistency. The system would allow the nonexpert or professional with limited background or knowledge of the sensory field to plan, execute, and report sensory results in a sound scientific manner following recommended practices. Knowledge, which might otherwise be lost or in a form too difficult or time consuming to use adequately, may be captured and stored within the system. The knowledge (sensory

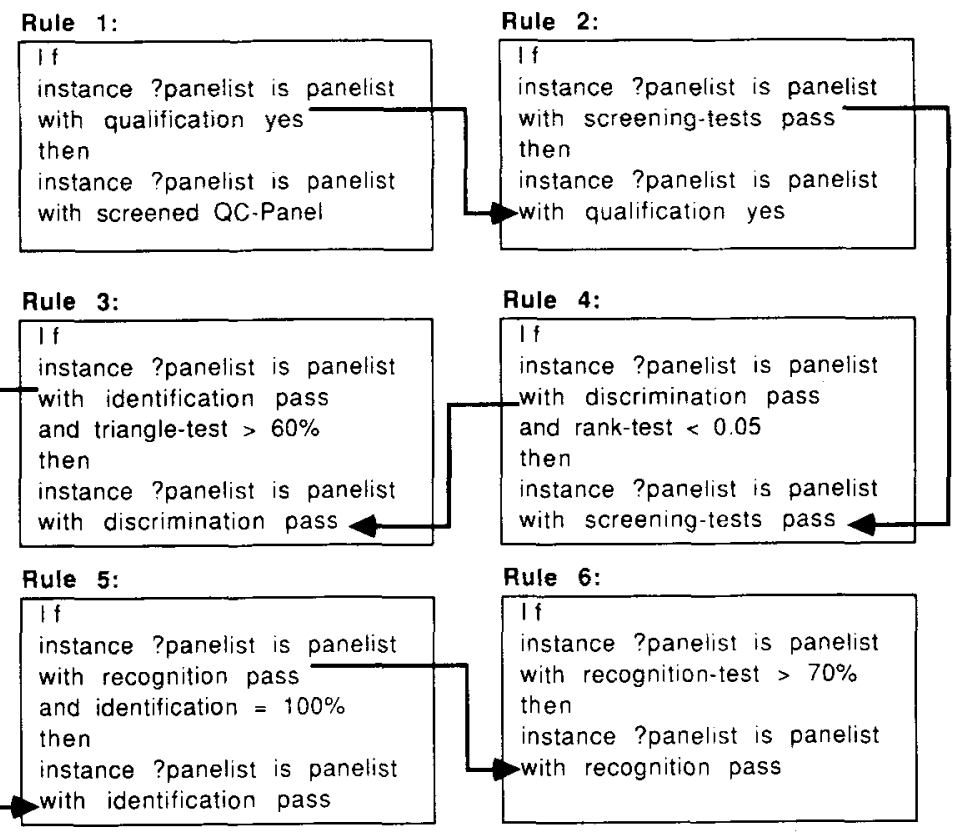

Figure 2. Illustration of backward chaining inferencing technique: Example rules for panelist screening. 


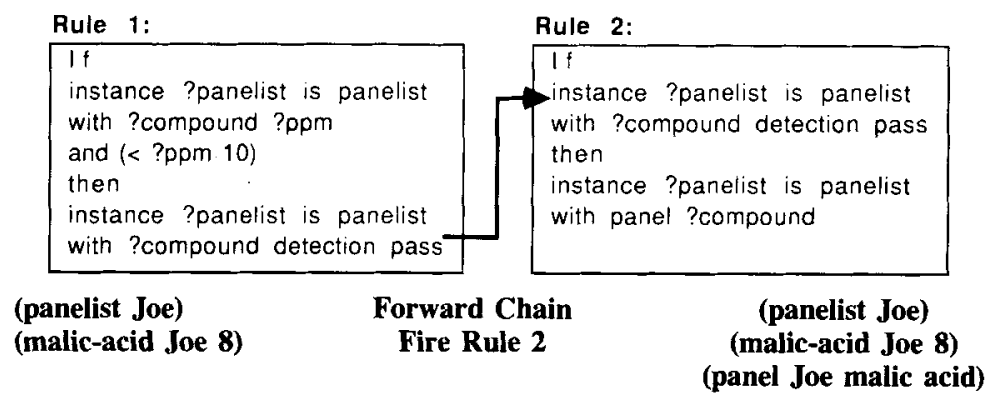

Figure 3. Illustration of forward chaining inferencing technique: Example rules for panelist selection.

methodology and statistical strategy) would be made available to a wider audience to communicate sensory testing methodology and promote the value of the sensory evaluation function.

Disadvantages that may be cited for the effort are as follows: (1) Development of an expert system is difficult and time consuming; most sensory professionals do not have the expertise or time to develop an expert system. (2) Hardware, software, development, and deliverance of a system of value demand both money and resources; although most sensory facilities have been computerized, few have devoted the resources to automate with expert systems. (3) Expert systems are not $100 \%$ reliable, perfect, or infallible; since business decisions are often based on sensory results, use and confidence in an expert system would be dependent on reliability. (4) Expert systems must be in a narrow domain of expertise; not only does the sensory domain cover many disciplines, the field is continuing to evolve. (5) Knowledge representation is limited; it is difficult to capture the "common sense" expertise sensory experts often rely on to conduct research. (6) A restricted, stylized language for input and output is required; sensory professionals would be required to learn the structure and syntax of yet another computer program. And (7) stylized and limited explanations of reasoning is required; sensory professionals would have to recognize and work within the limitations of what an expert system could deliver.

\section{Summary}

Sensory evaluation, an already computer-intensive discipline, is an area where expert systems can be developed, delivered, and welcomed by users. Thomson (1989) states that expert systems have an exciting future in sensory analysis, offering tremendous potential for education. Risvik and Rogers (1989) provide a discussion on the ultimate expert system for sensory evaluation. The users themselves must become demanding and actively involved in this development in order to obtain the most effective and productive expert system. Expert system technology is still a young field of endeavor and as such suffers from much misunderstanding and disappointment from overzealous expectations. With realistic expectations, we believe ex- pert system technology will serve sensory evaluation professionals by providing the opportunity to (1) formalize and clarify knowledge, (2) acquire and disseminate expertise, (3) provide computer-aided assistance, and (4) promote the value of sensory evaluation.

\section{REFERENCES}

ASTM СоммITTEE E-I8 (1981). Guidelines for the selection and training of sensory panel members (STP 758). Philadelphia: American Society for Testing and Materials.

BraUn, R. (1989). Expert system tools for knowledge analysis. AI Expert, 4(10), 22-24.

FrENZEL, L. E., JR. (1987). Crash course in artificial intelligence and expert systems (1st ed.). Indianapolis, IN: Howard W. Sams.

GALE, W. A. (1986). Artificial intelligence and statistics. Reading, MA: Wesley.

HARMON, P. (1987). Intelligent job aids: How AI will change training in the next five years. In G. P. Kearsley (Ed.), Artificial intelligence and instruction (pp. 165-177). Reading, MA: Addison-Wesley.

HARMON, P., \& KING, D. (1985). Expert systems: Artifical intelligence in business. New York: Wiley.

Hayes-Roth, F., Waterman, D. A., \& Lenat, D. B. (1983). Building expert systems. Reading, MA: Addison-Wesley.

KaHN, G., Nowlan, S., \& MCDermott, J. (1985). Strategies for knowledge acquisition. IEEE Transactions on Pattem Analysis \& Machine Intelligence, PAMI-7, 511-522.

LORENZEN, T. J., \& TRUSS, L. T. (1990). Anatomy of DEXPERT: An expert system for the design of experiments (Research Publication GMR-7111, pp. 1-30). Warren, MI: General Motors Research Laboratories.

MCLeLlaN, M. R. (1989). Introduction to artificial intelligence and expert systems. Food Technology, 5, 120-128.

NAES, T. (1990). Handling individual differences between assessors in sensory profiling. Food Quality \& Preference, 2, 187-199.

OLDFORD, W. (1987). Software abstraction of elements of statistical strategy (Personal communication). Preliminary papers of the Second International Workshop on Artificial Intelligence and Statistics, January 4-7, 1989. Fort Lauderdale, FL.

PARSAye, K., \& Chignell, M. (1988). Expert systems for experts. New York: Wiley.

Pregibon, D., \& Gale, W. A. (1984). REX: An expert system for Regression Analysis. (Statistical Research Rep. No. 4, pp. 1-10). Murray Hill, NJ.

Risvik, E., \& Rogers, R. (1989). Sensory analysis: A view on the use of computers. Food Quality \& Preference, 1, 81-85.

Thомson, D. M. H. (1989). Structuring the education of sensory analysts in the age of computers. Food Quality \& Preference, 1, 191-194

Waterman, D. A. (1985). A guide to expert systems (Reading, MA: Addison-Wesley. 